\title{
Ground State Solutions to a Critical Nonlocal Integrodifferential System
}

\author{
Min Liu ${ }^{\mathbb{D}}{ }^{1}{ }^{1}$ Zhijing Wang, ${ }^{1}$ and Zhenyu Guo $\mathbb{D}^{2}$ \\ ${ }^{1}$ School of Sciences, Liaoning Shihua University, Fushun 113001, China \\ ${ }^{2}$ School of Mathematics, Liaoning Normal University, Dalian 116029, China \\ Correspondence should be addressed to Zhenyu Guo; guozy@163.com
}

Received 30 April 2018; Accepted 18 July 2018; Published 8 August 2018

Academic Editor: Emmanuel Lorin

Copyright (c) 2018 Min Liu et al. This is an open access article distributed under the Creative Commons Attribution License, which permits unrestricted use, distribution, and reproduction in any medium, provided the original work is properly cited.

Consider the following nonlocal integrodifferential system: $\mathscr{L}_{K} u+\lambda_{1} u+\mu_{1}|u|^{2_{*}-2} u+G_{u}(x, u, v)=0$ in $\Omega, \mathscr{L}_{K} v+\lambda_{2} v+\mu_{2}|v|^{2_{*}-2} v+$ $G_{v}(x, u, v)=0$ in $\Omega, u=0, v=0$ in $\mathbb{R}^{N} \backslash \Omega$, where $\mathscr{L}_{K}$ is a general nonlocal integrodifferential operator, $\lambda_{1}, \lambda_{2}, \mu_{1}, \mu_{2}>0$, $2_{*}:=2 N /(N-2 s)$ is a fractional Sobolev critical exponent, $0<s<1, N>2 s, G(x, u, v)$ is a lower order perturbation of the critical coupling term, and $\Omega$ is an open bounded domain in $\mathbb{R}^{N}$ with Lipschitz boundary. Under proper conditions, we establish an existence result of the ground state solution to the nonlocal integrodifferential system.

\section{Introduction}

Recently, fractional Sobolev spaces and the corresponding nonlocal equations are applied to many subjects, such as, anomalous diffusion, elliptic problems with measure data, gradient potential theory, minimal surfaces, nonuniformly elliptic problems, optimization, phase transitions, quasigeostrophic flows, singular set of minima of variational functionals, and water waves (see [1] and the references therein). For more details, please refer the book [2]. In [3], the authors considered the following equation:

$$
\begin{aligned}
\mathscr{L}_{K} u+\lambda u+|u|^{2_{*}-2} u+f(x, u)=0 & \text { in } \Omega, \\
u=0 & \text { in } \mathbb{R}^{N} \backslash \Omega,
\end{aligned}
$$

where $\mathscr{L}_{K}$ is a general nonlocal integrodifferential operator of order $s$ which will be defined later and $f$ is a lower order perturbation of the critical power $|u|^{2_{*}-2} u$, and, under proper conditions, an existence result of its solutions was obtained.

In present paper, we use some ideas and techniques in [35] to extend the results in [3] to the system case.

Consider the following nonlocal integrodifferential system:

$$
\begin{array}{rr}
\mathscr{L}_{K} u+\lambda_{1} u+\mu_{1}|u|^{2_{*}-2} u+G_{u}(x, u, v)=0 & \text { in } \Omega, \\
\mathscr{L}_{K} v+\lambda_{2} v+\mu_{2}|v|^{2_{*}-2} v+G_{v}(x, u, v)=0 & \text { in } \Omega,
\end{array}
$$

$$
u=0, v=0
$$

$$
\text { in } \mathbb{R}^{N} \backslash \Omega \text {, }
$$

where $\lambda_{1}, \lambda_{2}, \mu_{1}, \mu_{2}>0,2_{*}:=2 N /(N-2 s)$ is a fractional Sobolev critical exponent, $0<s<1, N>2 s, G(x, u, v)$ is a lower order perturbation of the critical coupling term, $\Omega$ is an open bounded domain in $\mathbb{R}^{N}$ with Lipschitz boundary, and $\mathscr{L}_{K}$ is a general nonlocal integrodifferential operator defined as

$$
\begin{array}{r}
\mathscr{L}_{K} u(x) \\
=\int_{\mathbb{R}^{N}}(u(x+y)+u(x-y)-2 u(x)) K(y) \mathrm{d} y, \\
x \in \mathbb{R}^{N} .
\end{array}
$$

Here, $K: \mathbb{R}^{N} \backslash\{0\} \longrightarrow(0,+\infty)$ is a function satisfying

$m K \in L^{1}\left(\mathbb{R}^{N}\right)$, where $m(x)=\min \left\{1,|x|^{2}\right\}$,

$\exists \theta>0$ such that $K(x) \geq \frac{\theta}{|x|^{N+2 s}}$,

$\forall x \in \mathbb{R}^{N} \backslash\{0\}$,

$K(-x)=K(x)$ for any $x \in \mathbb{R}^{N} \backslash\{0\}$. 
Define the Hilbert space $D_{K}(\Omega)$ as the completion of $C_{c}^{\infty}(\Omega)$ with respect to the norm $\|\cdot\|_{D_{K}}$ induced by the scalar product $\langle\cdot, \cdot\rangle_{D_{K}}$ given by

$$
\begin{aligned}
& \langle u, v\rangle_{D_{K}}:=\int_{\mathbb{R}^{2 N}}(u(x)-u(y))(v(x)-v(y)) \\
& \cdot K(x-y) \mathrm{d} x \mathrm{~d} y .
\end{aligned}
$$

If $\Omega$ is an open bounded Lipschitz domain, then $D_{K}(\Omega)$ coincides with the Sobolev space

$$
X_{0}:=\left\{f \in X: f=0 \text { a.e. in } \Omega^{c}\right\},
$$

where $X$ is a linear space of Lebesgue measurable functions from $\mathbb{R}^{N}$ to $\mathbb{R}$ such that the restriction to $\Omega$ of any function $f$ in $X$ belongs to $L^{2}(\Omega)$ and the map $(x, y) \longmapsto(f(x)-$ $f(y)) \sqrt{K(x-y)}$ is in $L^{2}\left(\mathbb{R}^{2 N} \backslash\left(\Omega^{c} \times \Omega^{c}\right), \mathrm{d} x \mathrm{~d} y\right)$, and $\Omega^{c}$ is the complement of $\Omega$ in $\mathbb{R}^{N}$.

The solutions of (2) coincide with the critical points of the following energy functional $E_{K}: \mathscr{D}_{K} \rightarrow \mathbb{R}$ :

$$
\begin{aligned}
E_{K}(u, v)= & \frac{1}{2}\|(u, v)\|_{\mathscr{D}_{K}}^{2}-\frac{1}{2}\left(\lambda_{1}|u|_{2, \Omega}^{2}+\lambda_{2}|v|_{2, \Omega}^{2}\right) \\
& -\frac{1}{2_{*}}\left(\mu_{1}|u|_{2_{*}^{*}, \Omega}^{2_{*}}+\mu_{2}|v|_{2_{*}^{*}, \Omega}^{2_{*}}\right) \\
& -\int_{\Omega} G(x, u(x), v(x)) \mathrm{d} x,
\end{aligned}
$$

where $|u|_{p, \Omega}=\left(\int_{\Omega}|u|^{p} \mathrm{~d} x\right)^{1 / p}, 1 \leq p<+\infty, \mathscr{D}_{K}(\Omega):=$ $D_{K}(\Omega) \times D_{K}(\Omega)$, endowed with norm $\|(u, v)\|_{D_{K}}^{2}:=\|u\|_{D_{K}}^{2}+$ $\|v\|_{D_{K}}^{2}$, and $G(x, s, t)$ is a nonnegative Carathéodory function from $\Omega \times \mathbb{R} \times \mathbb{R}$ to $\mathbb{R}$; namely,

$$
\begin{aligned}
& \forall \delta>0, \sup \left\{\frac{|G(x, s, t)|}{|s|+|t|}: \text { a.e. } x \in \Omega,|(s, t)|\right. \\
& \left.:=\sqrt{s^{2}+t^{2}} \leq \delta\right\}<+\infty, \\
& \lim _{(s, t) \longrightarrow(0,0)} \frac{G(x, s, t)}{|s|^{2}+|t|^{2}}=0 \quad \text { uniformly in } x \in \Omega, \\
& \lim _{|(s, t)| \longrightarrow+\infty} \frac{G(x, s, t)}{|s|^{2_{*}}+|t|^{2_{*}}}=0 \quad \text { uniformly in } x \in \Omega .
\end{aligned}
$$

Consider the following Nehari manifold:

$$
\begin{aligned}
\mathscr{M}_{K} & :=\left\{(u, v) \in \mathscr{D}_{K}(\Omega) \backslash\{(0,0)\}:\|(u, v)\|_{\mathscr{D}_{K}}^{2}\right. \\
& =\lambda_{1}|u|_{2, \Omega}^{2}+\lambda_{2}|v|_{2, \Omega}^{2}+\mu_{1}|u|_{2_{*}^{*}, \Omega}^{2_{*}}+\mu_{2}|v|_{2_{*}, \Omega}^{2_{*}} \\
& +\int_{\Omega}\left(G_{u}(x, u(x), v(x)) u(x)\right. \\
& \left.\left.+G_{v}(x, u(x), v(x)) v(x)\right) \mathrm{d} x\right\},
\end{aligned}
$$

define the ground state energy of (2) by

$$
B_{K}:=\inf _{(u, v) \in \mathscr{M}_{K}} E_{K}(u, v),
$$

and call a solution $(u, v)$ by a ground state solution if $E_{K}(u, v)=B_{K}$. Define

$$
\begin{gathered}
\bar{S}_{K}:=\inf _{\substack{(u, v) \in \mathscr{D}_{K}\left(\mathbb{R}^{N}\right) \\
(u, v) \neq(0,0)}} \frac{\|(u, v)\|_{\mathscr{D}_{K}}^{2}}{\left(\mu_{1}|u|_{2_{*}, \mathbb{R}^{N}}^{2_{*}}+\mu_{2}|v|_{2_{*}, \mathbb{R}^{N}}^{2_{*}}\right)^{2 / 2_{*}}}, \\
\bar{S}_{K, \lambda_{1}, \lambda_{2}} \\
:=\inf _{\substack{(u, v) \in \mathscr{D}_{K}(\Omega) \\
(u, v) \neq(0,0)}} \frac{\|(u, v)\|_{\mathscr{D}_{K}}^{2}-\lambda_{1}|u|_{2, \Omega}^{2}-\lambda_{2}|v|_{2, \Omega}^{2}}{\left(\mu_{1}|u|_{2_{*}, \Omega}^{2_{*}}+\mu_{2}|v|_{2_{*}, \Omega}^{2_{*}}\right)^{2 / 2_{*}}} .
\end{gathered}
$$

Then $\bar{S}_{K, \lambda_{1}, \lambda_{2}} \leq \min \left\{\bar{S}_{K}, S_{K, \lambda_{1}}, S_{K, \lambda_{2}}\right\}$, where

$$
\begin{aligned}
S_{K, \lambda_{1}} & :=\inf _{\substack{u \in D_{K}(\Omega) \\
u \neq 0}} \frac{\|u\|_{D_{K}}^{2}-\lambda_{1}|u|_{2, \Omega}^{2}}{\left(\mu_{1}|u|_{2_{*}, \Omega}^{2_{*}}\right)^{2 / 2_{*}}}, \\
S_{K, \lambda_{2}} & :=\inf _{\substack{v \in D_{K}(\Omega) \\
v \neq 0}} \frac{\|v\|_{D_{K}}^{2}-\lambda_{2}|v|_{2, \Omega}^{2}}{\left(\mu_{2}|v|_{2_{*}, \Omega}^{2_{*}}\right)^{2 / 2_{*}}} .
\end{aligned}
$$

Theorem 1. Assume that $\lambda_{1}, \lambda_{2} \in\left(0, \lambda_{K}(\Omega)\right)$, where $\lambda_{K}(\Omega)$ is the first eigenvalue of the nonlocal operator $-\mathscr{L}_{K}$ with homogeneous Dirichlet boundary conditions. If there exists $\left(u_{0}, v_{0}\right) \in \mathscr{D}_{K}(\Omega) \backslash\{(0,0)\}$ with $u_{0}, v_{0} \geq 0$ a.e. in $\mathbb{R}^{N}$, such that

$$
\sup _{t>0} E_{K}\left(t u_{0}, t v_{0}\right)<\frac{s}{N} \bar{S}_{K, \lambda_{1}, \lambda_{2}}^{N / 2 s},
$$

then (2) has a nontrivial ground state solution.

\section{Preliminaries}

We need the following results, which have been proved in [6].

Lemma 2. There exists a positive constant $C_{s}$ such that, for any $u \in D_{K}(\Omega)$,

$$
C_{s} \int_{\mathbb{R}^{2 N}} \frac{|u(x)-u(y)|^{2}}{|x-y|^{N+2 s}} \mathrm{~d} x \mathrm{~d} y \geq|u|_{2_{*}, \mathbb{R}^{N}}^{2}=|u|_{2_{*}, \Omega}^{2},
$$

where $C_{s}$ is only depending on $N$ and $s$.

\section{Proof of Theorem 1}

Lemma 3. Assume that $G: \Omega \times \mathbb{R} \times \mathbb{R} \longrightarrow \mathbb{R}$ is a Carathéodory function; that is, (10)-(12) hold. Then, for any $\varepsilon>0$, there exist $a(\varepsilon)>0$ and $b(\varepsilon)>0$ such that for a.e. $x \in \Omega$ and, for any $s, t \in \mathbb{R}$,

$$
\begin{aligned}
& |G(x, s, t)| \leq \varepsilon\left(|s|^{2_{*}}+|t|^{2_{*}}\right)+a(\varepsilon)(|s|+|t|), \\
& |G(x, s, t)| \leq \varepsilon\left(|s|^{2}+|t|^{2}\right)+b(\varepsilon)\left(|s|^{2_{*}}+|t|^{2_{*}}\right) .
\end{aligned}
$$

Proof. For any $\varepsilon>0$, by (12), there exists $\delta=\delta(\varepsilon)>0$ such that, for a.e. $x \in \Omega$ and for any $(s, t) \in \mathbb{R}^{2}$ with $|(s, t)|>\delta$,

$$
|G(x, s, t)| \leq \varepsilon\left(|s|^{2_{*}}+|t|^{2_{*}}\right) .
$$


It follows from (10) that there exists $a(\varepsilon)=a(\delta(\varepsilon))>0$ satisfying, for a.e. $x \in \Omega$ and for any $(s, t) \in \mathbb{R} \times \mathbb{R}$ with $|(s, t)| \leq \delta$,

$$
|G(x, s, t)| \leq a(\varepsilon)(|s|+|t|) .
$$

Combining (21) and (22), we see that (19) holds.

By (11), for any $\varepsilon>0$, there exists $\sigma=\sigma(\varepsilon)>0$ such that, for a.e. $x \in \Omega$ and for any $(s, t) \in \mathbb{R}^{2}$ with $|(s, t)|<\sigma$,

$$
|G(x, s, t)| \leq \varepsilon\left(|s|^{2}+|t|^{2}\right) .
$$

It is easy to see from (19) that

$$
\begin{aligned}
|G(x, s, t)| & \leq|s|^{2_{*}}+|t|^{2_{*}}+a(1)(|s|+|t|) \\
& =\left(|s|^{2_{*}}+|t|^{2_{*}}\right)\left(1+\frac{a(1)(|s|+|t|)}{|s|^{2_{*}}+|t|^{2_{*}}}\right) .
\end{aligned}
$$

Therefore, there exists $b(\varepsilon)=b(\sigma(\varepsilon))>0$ satisfying, for a.e. $x \in \Omega$ and for any $(s, t) \in \mathbb{R}^{2}$ with $|(s, t)| \geq \sigma$,

$$
|G(x, s, t)| \leq b(\varepsilon)\left(|s|^{2_{*}}+|t|^{2_{*}}\right) .
$$

Then, (20) follows from (23) and (25).

Lemma 4. Assume that $\lambda_{1}, \lambda_{2} \in\left(0, \lambda_{K}(\Omega)\right)$. Then, there exist $\rho, \tau>0$ and $e=\left(e_{1}, e_{2}\right) \in \mathscr{D}_{K}(\Omega)$ such that

(i) $E_{K}(u, v) \geq \tau$ for any $(u, v) \in \mathscr{D}_{K}(\Omega)$ with $\|(u, v)\|_{\mathscr{D}_{K}}=$ $\rho$;

(ii) $e_{1}, e_{2} \geq 0$ a.e. in $\mathbb{R}^{N},\left\|\left(e_{1}, e_{2}\right)\right\|_{\mathscr{D}_{K}}>\rho$, and $E_{K}\left(e_{1}\right.$, $\left.e_{2}\right)<\tau$.

In particular, if (17) holds, then we can construct e by e = $t_{0}\left(u_{0}, v_{0}\right)$, where $\left(u_{0}, v_{0}\right)$ is given by (17) and $t_{0}>0$ is large enough.

Proof. For $(u, v) \in \mathscr{D}_{K}(\Omega)$ and any $\varepsilon>0$, by (20), Lemma 2, $H^{s}\left(\mathbb{R}^{N}\right) \hookrightarrow L^{2_{*}}\left(\mathbb{R}^{N}\right)$, and (5), we deduce that

$$
\begin{gathered}
E_{K}(u, v) \geq \frac{1}{2}\|u, v\|_{\mathscr{D}_{K}}^{2}-\frac{1}{2}\left(\lambda_{1}|u|_{2, \Omega}^{2}+\lambda_{2}|v|_{2, \Omega}^{2}\right) \\
-\frac{1}{2_{*}}\left(\mu_{1}|u|_{2_{*}, \Omega}^{2_{*}}+\mu_{2}|v|_{2_{*}, \Omega}^{2_{*}}\right) \\
-\int_{\Omega} \varepsilon\left[\left(u^{2}+v^{2}\right)+b(\varepsilon)\left(|u|^{2_{*}}+|v|^{2 *}\right)\right] \mathrm{d} x \\
\quad \geq \frac{1}{2}\left(1-\frac{\lambda_{1}}{\lambda_{K}(\Omega)}\right)\|u\|_{D_{K}}^{2}+\frac{1}{2}\left(1-\frac{\lambda_{2}}{\lambda_{K}(\Omega)}\right) \\
+\|v\|_{D_{K}}^{2}-\varepsilon|\Omega|^{\left(2_{*}-2\right) / 2_{*}}\left(|u|_{2_{*}, \Omega}^{2}+|v|_{2_{*}, \Omega}^{2}\right)-\left(\frac{\mu_{1}}{2_{*}}\right. \\
+b(\varepsilon))|u|_{2_{*}, \Omega}^{2_{*}}-\left(\frac{\mu_{2}}{2_{*}}+b(\varepsilon)\right)|v|_{2_{*}, \Omega}^{2_{*}} \geq \frac{1}{2}(1 \\
\left.-\frac{\lambda_{1}}{\lambda_{K}(\Omega)}\right)\|u\|_{D_{K}}^{2}+\frac{1}{2}\left(1-\frac{\lambda_{2}}{\lambda_{K}(\Omega)}\right)\|v\|_{D_{K}}^{2} \\
-\varepsilon|\Omega|^{\left(2_{*}-2\right) / 2_{*}} C_{s}\left(\int_{\mathbb{R}^{2 N}} \frac{|u(x)-u(y)|^{2}}{|x-y|^{N+2 s}} \mathrm{~d} x \mathrm{~d} y\right.
\end{gathered}
$$

$$
\begin{aligned}
& \left.+\int_{\mathbb{R}^{2 N}} \frac{|v(x)-v(y)|^{2}}{|x-y|^{N+2 s}} \mathrm{~d} x \mathrm{~d} y\right)-\left(\frac{\mu_{1}}{2_{*}}+b(\varepsilon)\right) \\
& \cdot C_{s}^{2_{*} / 2}\left(\int_{\mathbb{R}^{2 N}} \frac{|u(x)-u(y)|^{2}}{|x-y|^{N+2 s}} \mathrm{~d} x \mathrm{~d} y\right)^{2_{*} / 2}-\left(\frac{\mu_{2}}{2_{*}}\right. \\
& +b(\varepsilon)) C_{s}^{2_{*} / 2}\left(\int_{\mathbb{R}^{2 N}} \frac{|v(x)-v(y)|^{2}}{|x-y|^{N+2 s}} \mathrm{~d} x \mathrm{~d} y\right)^{2_{*} / 2} \\
& \geq\left[\frac{1}{2}\left(1-\frac{\lambda_{1}}{\lambda_{K}(\Omega)}\right)-\frac{\varepsilon C_{s}|\Omega|^{\left(2_{*}-2\right) / 2_{*}}}{\theta}\right]\|u\|_{D_{K}}^{2} \\
& +\left[\frac{1}{2}\left(1-\frac{\lambda_{2}}{\lambda_{K}(\Omega)}\right)-\frac{\varepsilon C_{s}|\Omega|^{\left(2_{*}-2\right) / 2_{*}}}{\theta}\right]\|v\|_{D_{K}}^{2} \\
& -\left(\frac{\mu_{1}}{2_{*}}+b(\varepsilon)\right)\left(\frac{C_{s}}{\theta}\right)^{2_{*} / 2}\|u\|_{D_{K}}^{2_{*}}-\left(\frac{\mu_{2}}{2_{*}}+b(\varepsilon)\right) \\
& \cdot\left(\frac{C_{s}}{\theta}\right)^{2_{*} / 2}\|v\|_{D_{K}}^{2_{*}} \cdot
\end{aligned}
$$

For $\varepsilon<\theta\left(\lambda_{K}(\Omega)-\max \left\{\lambda_{1}, \lambda_{2}\right\}\right) / 2 C_{s} \lambda_{K}(\Omega)|\Omega|^{\left(2_{*}-2\right) / 2_{*}}$, we have

$$
E_{K}(u, v) \geq a\|(u, v)\|_{\mathscr{D}_{K}}^{2}\left(1-b\|(u, v)\|_{\mathscr{D}_{K}}^{2_{*}-2}\right),
$$

where $a$ and $b$ are suitable positive constants. Let $(u, v) \in \mathscr{D}_{K}$ satisfy $\|u, v\|_{\mathscr{D}_{K}}=\rho>0$, where $\rho$ is small enough such that $1-b \rho^{2 *-2}>0$. Therefore,

$$
\inf _{\substack{(u, v) \in \mathscr{D}_{K}(\Omega) \\\|(u, v)\|_{\mathscr{D}_{K}}=\rho}} E_{K}(u, v) \geq a \rho^{2}\left(1-b \rho^{2_{*}-2}\right):=\tau>0
$$

which proves (i).

Fix $(u, v) \in \mathscr{D}_{K}(\Omega)$ with $\|(u, v)\|_{\mathscr{D}_{K}}=1$ and $u \geq 0, v \geq 0$ a.e. in $\mathbb{R}^{N}$. Choosing $\varepsilon=\left(\min \left\{\mu_{1}, \mu_{2}\right\}\right) /\left(2 \cdot 2_{*}\right)$ in (19), we get that

$$
\begin{aligned}
|G(x, u, v)| \leq & \frac{\min \left\{\mu_{1}, \mu_{2}\right\}}{2 \cdot 2_{*}}\left(|u|^{2_{*}}+|v|^{2_{*}}\right) \\
& +a\left(\frac{\min \left\{\mu_{1}, \mu_{2}\right\}}{2 \cdot 2_{*}}\right)(|u|+|v|) .
\end{aligned}
$$

For $t>0$, by (29), we have

$$
\begin{aligned}
E_{K}(t u, t v)= & \frac{t^{2}}{2}\|u, v\|_{\mathscr{D}_{K}}^{2}-\frac{t^{2}}{2}\left(\lambda_{1}|u|_{2, \Omega}^{2}+\lambda_{2}|v|_{2, \Omega}^{2}\right) \\
& -\frac{t^{2 *}}{2_{*}}\left(\mu_{1}|u|_{2_{*}, \Omega}^{2_{*}}+\mu_{2}|v|_{2_{*}, \Omega}^{2_{*}}\right) \\
& -\int_{\Omega} G(x, t u, t v) \mathrm{d} x \\
\leq & \frac{t^{2}}{2}-\frac{t^{2_{*}} \min \left\{\mu_{1}, \mu_{2}\right\}}{2_{*}}\left(|u|_{2_{*}, \Omega}^{2_{*}}+|v|_{2_{*}, \Omega}^{2_{*}}\right)
\end{aligned}
$$




$$
\begin{aligned}
& +\frac{t^{2_{*}} \min \left\{\mu_{1}, \mu_{2}\right\}}{2 \cdot 2_{*}} \int_{\Omega}\left(|u|^{2_{*}}+|v|^{2_{*}}\right) \mathrm{d} x \\
& +\operatorname{ta}\left(\frac{\min \left\{\mu_{1}, \mu_{2}\right\}}{2 \cdot 2_{*}}\right) \int_{\Omega}(|u|+|v|) \mathrm{d} x \\
& \leq \frac{t^{2}}{2}-\frac{\min \left\{\mu_{1}, \mu_{2}\right\}}{2 \cdot 2_{*}}\left(|u|_{2_{*}, \Omega}^{2_{*}}+|v|_{2_{*}, \Omega}^{2_{*}}\right) t^{2_{*}} \\
& +a\left(\frac{\min \left\{\mu_{1}, \mu_{2}\right\}}{2 \cdot 2_{*}}\right)\left(|u|_{2, \Omega}^{2}+|v|_{2, \Omega}^{2}\right) t,
\end{aligned}
$$

which implies that $E_{K}(t u, t v) \longrightarrow-\infty$ as $t \longrightarrow+\infty$. Then, (ii) follows by taking $e=\left(e_{1}, e_{2}\right)=(t u, t v)$ with $t$ sufficiently large.

In particular, if (17) holds, then we may fix $(u, v)$ by $(u, v)=\left(u_{0}, v_{0}\right) /\left\|\left(u_{0}, v_{0}\right)\right\|_{\mathscr{D}_{K}}$. Thus, we can construct $e$ by $e=\left(t u_{0}, t v_{0}\right) /\left\|u_{0}, v_{0}\right\|_{\mathscr{D}_{K}}=t_{0}\left(u_{0}, v_{0}\right)$, where $t_{0}$ is large enough.

Define

$$
\mathscr{B}_{K}:=\inf _{h \in \Gamma} \sup _{0<t<1} E_{K}(h(t))
$$

where

$$
\begin{aligned}
\Gamma:= & \left\{h \in C\left([0,1], \mathscr{D}_{K}(\Omega)\right): h(0)=0, h(1)=e\right. \\
& \left.=\left(e_{1}, e_{2}\right)\right\}
\end{aligned}
$$

with $e=t_{0}\left(u_{0}, v_{0}\right)$ given in Lemma 4 . It is standard to see that

$$
\mathscr{B}_{K}=\mathbb{B}_{K}=B_{K}
$$

where $\mathbb{B}_{K}=\inf _{(u, v) \in \mathscr{D}_{K}(\Omega) \backslash\{(0,0)\}} \sup _{t>0} E_{K}(t u, t v)$ and $B_{K}$ is given in (14).

Lemma 5. $\tau \leq B_{K}<(s / N) \bar{S}_{K, \lambda_{1}, \lambda_{2}}^{N / 2 s}$, where $\tau$ is given in Lemma 4 and $\bar{S}_{K, \lambda_{1}, \lambda_{2}}$ is defined in (15).

Proof. Noting that, for any $h \in \Gamma$, the function $t \longmapsto\|h(t)\|_{\mathscr{D}_{K}}$ is continuous in $[0,1]$; we get that $\|h(0)\|_{\mathscr{D}_{K}}=\|(0,0)\|_{\mathscr{D}_{K}}=$ $0<\rho$ and $\|h(1)\|_{D_{K}}=\|e\|_{\mathscr{D}_{K}}>\rho$, where $\rho$ is given by Lemma 4 . Thus, we may find $t_{h} \in(0,1)$ such that $\left\|h\left(t_{h}\right)\right\|_{\mathscr{D}_{K}}=$ $\rho$. Therefore,

$$
\begin{aligned}
\sup _{0<t<1} E_{K}(h(t)) & \geq E_{K}\left(h\left(t_{h}\right)\right) \geq \inf _{\substack{(u, v) \in \mathscr{D}_{K}(\Omega) \\
\|(u, v)\|_{\mathscr{D}_{K}}=\rho}} E_{K}(u, v) \\
& \geq \tau
\end{aligned}
$$

which implies that $B_{K} \geq \tau$. Recalling that $e=t_{0}\left(u_{0}, v_{0}\right)$ and the special pass $[0,1] \ni t \longmapsto t t_{0}\left(u_{0}, v_{0}\right)$ belongs to $\Gamma$, by (17), we deduce that

$$
\begin{aligned}
B_{K} & =\inf _{h \in \Gamma_{0<t<1}} E_{K}(h(t)) \leq \sup _{t>0} E_{K}\left(t u_{0}, t v_{0}\right) \\
& <\frac{s}{N} S_{K, \lambda_{1}, \lambda_{2}}^{N / 2 s} .
\end{aligned}
$$

Lemma 6. Every sequence $\left\{\left(u_{n}, v_{n}\right)\right\}$ with $E_{K}\left(u_{n}, v_{n}\right) \rightarrow c$ and $E_{K}^{\prime}\left(u_{n}, v_{n}\right) \longrightarrow 0$ in $\mathscr{D}_{K}^{\prime}(\Omega)$ as $n \longrightarrow \infty\left((P S)_{c}\right.$ sequence of $\left.E_{K}\right)$ is bounded in $\mathscr{D}_{K}(\Omega)$.

Proof. From

$$
\begin{aligned}
c+o(1)= & E_{K}\left(u_{n}, v_{n}\right) \\
= & \frac{1}{2}\left\|\left(u_{n}, v_{n}\right)\right\|_{\mathscr{D}_{K}}^{2} \\
& -\frac{1}{2}\left(\lambda_{1}\left|u_{n}\right|_{2, \Omega}^{2}+\lambda_{2}\left|v_{n}\right|_{2, \Omega}^{2}\right) \\
& -\frac{1}{2_{*}}\left(\mu_{1}\left|u_{n}\right|_{2_{*}, \Omega}^{2_{*}}+\mu_{2}\left|v_{n}\right|_{2_{*}, \Omega}^{2_{*}}\right) \\
& -\int_{\Omega} G\left(x, u_{n}, v_{n}\right) \mathrm{d} x
\end{aligned}
$$

and

$$
\begin{aligned}
o(1)= & E_{K}^{\prime}\left(u_{n}, v_{n}\right)\left(u_{n}, v_{n}\right) \\
= & \left\|\left(u_{n}, v_{n}\right)\right\|_{\mathscr{D}_{K}}^{2}-\left(\lambda_{1}\left|u_{n}\right|_{2, \Omega}^{2}+\lambda_{2}\left|v_{n}\right|_{2, \Omega}^{2}\right) \\
& -\left(\mu_{1}\left|u_{n}\right|_{2_{*}^{*}, \Omega}^{2_{*}}+\mu_{2}\left|v_{n}\right|_{2_{*}, \Omega}^{2_{*}}\right) \\
& -\int_{\Omega}\left(G_{u}\left(x, u_{n}, v_{n}\right) u_{n}+G_{v}\left(x, u_{n}, v_{n}\right) v_{n}\right) \mathrm{d} x,
\end{aligned}
$$

it can be seen that

$$
\begin{aligned}
& c+o(1)=E_{K}\left(u_{n}, v_{n}\right)-\frac{1}{2} E_{K}^{\prime}\left(u_{n}, v_{n}\right)\left(u_{n}, v_{n}\right) \\
&=\left(\frac{1}{2}-\frac{1}{2_{*}}\right)\left(\mu_{1}\left|u_{n}\right|_{2_{*}, \Omega}^{2_{*}}+\mu_{2}\left|v_{n}\right|_{2_{*}^{*}, \Omega}^{2_{*}}\right) \\
& \quad-\int_{\Omega} G\left(x, u_{n}, v_{n}\right) \mathrm{d} x \\
&+\frac{1}{2} \int_{\Omega}\left(G_{u}\left(x, u_{n}, v_{n}\right) u_{n}+G_{v}\left(x, u_{n}, v_{n}\right) v_{n}\right) \mathrm{d} x \\
& \geq \frac{s}{N}\left(\mu_{1}\left|u_{n}\right|_{2_{*}, \Omega}^{2_{*}}+\mu_{2}\left|v_{n}\right|_{2_{*}, \Omega}^{2_{*}}\right) \\
&-k \int_{\Omega}\left|G\left(x, u_{n}, v_{n}\right)\right| \mathrm{d} x
\end{aligned}
$$

for some suitable positive constant $k$. For $u \in D_{K}(u)$, it follows from Lemma 2 and (5) that

$$
\begin{aligned}
& \int_{\Omega}|u| \mathrm{d} x \leq|\Omega|^{\left(2_{*}-1\right) / 2_{*}}|u|_{2_{*}, \Omega} \\
& \quad \leq|\Omega|^{\left(2_{*}-1\right) / 2_{*}}\left(C_{s} \int_{\mathbb{R}^{2 N}} \frac{|u(x)-u(y)|^{2}}{|x-y|^{N+2 s}} \mathrm{~d} x \mathrm{~d} y\right)^{1 / 2} \\
& \leq \Theta\|u\|_{D_{K}}
\end{aligned}
$$


for some positive constant $\Theta$. By (19) and (39), we have

$$
\begin{aligned}
\int_{\Omega}\left|G\left(x, u_{n}, v_{n}\right)\right| \mathrm{d} x \leq & \varepsilon\left(\left|u_{n}\right|_{2_{*}, \Omega}^{2_{*}}+\left|v_{n}\right|_{2_{*}, \Omega}^{2_{*}}\right) \\
& +a(\varepsilon) \Theta\left(\left\|u_{n}\right\|_{D_{K}}+\left\|v_{n}\right\|_{D_{K}}\right) \\
\leq & \varepsilon\left(\left|u_{n}\right|_{2_{*}^{*}, \Omega}^{2_{*}}+\left|v_{n}\right|_{2_{*}, \Omega}^{2_{*}}\right) \\
& +C(\varepsilon)\left\|\left(u_{n}, v_{n}\right)\right\|_{D_{K}},
\end{aligned}
$$

where $C(\varepsilon)$ is a positive constant relevant to $\varepsilon$ and $\Omega$. Choosing $\varepsilon$ small enough such that $(s / N) \min \left\{\mu_{1}, \mu_{2}\right\}-k \varepsilon>$ 0 , then (38) and (40) yield that

$$
\left|u_{n}\right|_{2_{*}, \Omega}^{2_{*}}+\left|v_{n}\right|_{2_{*}, \Omega}^{2_{*}} \leq C\left(1+\left\|\left(u_{n}, v_{n}\right)\right\|_{\mathscr{D}_{K}}\right) .
$$

Letting $\varepsilon=1$ in (40) and noting $0<\lambda_{1}, \lambda_{2}<\lambda_{K}(\Omega)$, by (41), we infer that

$$
\begin{aligned}
c+o(1)=E_{K}\left(u_{n}, v_{n}\right) & \frac{1}{2}\left(1-\frac{\lambda_{1}}{\lambda_{K}(\Omega)}\right)\left\|u_{n}\right\|_{D_{K}}^{2} \\
& +\frac{1}{2}\left(1-\frac{\lambda_{2}}{\lambda_{K}(\Omega)}\right)\left\|v_{n}\right\|_{D_{K}}^{2} \\
& -\frac{1}{2_{*}}\left(\mu_{1}\left|u_{n}\right|_{2_{*}^{*}, \Omega}^{2_{*}}+\mu_{2}\left|v_{n}\right|_{2_{*}, \Omega}^{2_{*}}\right) \\
& -\int_{\Omega}\left|G\left(x, u_{n}, v_{n}\right)\right| \mathrm{d} x \\
\geq & \frac{1}{2}\left(1-\frac{\max \left\{\lambda_{1}, \lambda_{2}\right\}}{\lambda_{K}(\Omega)}\right)\left\|\left(u_{n}, v_{n}\right)\right\|_{\mathscr{D}_{K}}^{2} \\
& -\left(\frac{\max \left\{\mu_{1}, \mu_{2}\right\}}{2_{*}}+1\right)\left(\left|u_{n}\right|_{2_{*}, \Omega}^{2_{*}}+\left|v_{n}\right|_{2_{*}, \Omega}^{2_{*}}\right) \\
& -C(1)\left\|\left(u_{n}, v_{n}\right)\right\|_{\mathscr{D}_{K}} \\
\geq & C_{1}\left\|\left(u_{n}, v_{n}\right)\right\|_{\mathscr{D}_{K}}^{2}-C_{2}\left(1+\left\|\left(u_{n}, v_{n}\right)\right\|_{\mathscr{D}_{K}}\right)
\end{aligned}
$$

for suitable constants $C_{1}$ and $C_{2}$, which implies that $\left\{\left(u_{n}, v_{n}\right)\right\}$ is bounded in $\mathscr{D}_{K}(\Omega)$.

Proof of Theorem 1. It follows from $G(x, 0,0)=0$ that $E_{K}(0,0)=0<\tau$, where $\tau$ is given in Lemma 4. By Lemma 4, we see that $E_{K}$ has a Mountain Pass Structure (e.g., [7]). By (33) as well as Lemma 5 and Theorem 2.2 in [8], there is $(P S)_{B_{K}}$ sequence $\left\{\left(u_{n}, v_{n}\right)\right\}$ for $E_{K}$. By Lemma 6, we get that $\left\{\left(u_{n}, v_{n}\right)\right\}$ is bounded in $\mathscr{D}_{K}(\Omega)$, and then, going if necessary to a subsequence, still denoted by $\left\{\left(u_{n}, v_{n}\right)\right\}$, there exists $\left(u_{\infty}, v_{\infty}\right) \in \mathscr{D}_{K}(\Omega)$ such that $\left(u_{n}, v_{n}\right) \rightarrow\left(u_{\infty}, v_{\infty}\right)$ weakly in $\mathscr{D}_{K}(\Omega)$; i.e.,

$$
\begin{aligned}
& \int_{\mathbb{R}^{2 N}}\left(u_{n}(x)-u_{n}(y)\right)(\phi(x)-\phi(y)) \\
& \cdot K(x-y) \mathrm{d} x \mathrm{~d} y+\int_{\mathbb{R}^{2 N}}\left(v_{n}(x)-v_{n}(y)\right)
\end{aligned}
$$

$$
\begin{gathered}
\cdot(\psi(x)-\psi(y)) K(x-y) \mathrm{d} x \mathrm{~d} y \longrightarrow \\
\int_{\mathbb{R}^{2 N}}\left(u_{\infty}(x)-u_{\infty}(y)\right)(\phi(x)-\phi(y)) \\
\cdot K(x-y) \mathrm{d} x \mathrm{~d} y+\int_{\mathbb{R}^{2 N}}\left(v_{\infty}(x)-v_{\infty}(y)\right) \\
\cdot(\psi(x)-\psi(y)) K(x-y) \mathrm{d} x \mathrm{~d} y
\end{gathered}
$$

for any $(\phi, \psi) \in \mathscr{D}_{K}(\Omega)$. It follows from (41) and the boundedness of $\left\{\left(u_{n}, v_{n}\right)\right\}$ in $\mathscr{D}_{K}(\Omega)$ that

$$
\left\{u_{n}\right\} \text { and }\left\{v_{n}\right\} \text { are bounded in } L^{2_{*}}(\Omega) \text {. }
$$

Then, it is standard to see that

$$
\begin{aligned}
& u_{n} \rightarrow u_{\infty}, \\
& v_{n} \rightarrow v_{\infty}
\end{aligned}
$$

weakly in $L^{2 *}(\Omega)$,

$$
\begin{aligned}
& u_{n} \longrightarrow u_{\infty} \\
& v_{n} \longrightarrow v_{\infty}
\end{aligned}
$$

strongly in $L^{p}(\Omega)$,

$$
\begin{aligned}
& u_{n} \longrightarrow u_{\infty} \\
& v_{n} \longrightarrow v_{\infty}
\end{aligned}
$$

a.e. in $\Omega$,

$$
\begin{gathered}
\left|u_{n}\right|^{2_{*}-2} u_{n} \rightarrow\left|u_{\infty}\right|^{2_{*}-2} u_{\infty}, \\
\left|v_{n}\right|^{2_{*}-2} v_{n} \rightarrow\left|v_{\infty}\right|^{2_{*}-2} v_{\infty}
\end{gathered}
$$

weakly in $L^{2 * /(2 *-1)}(\Omega)$,

up to a subsequence, where $p \in\left[1,2_{*}\right)$. By (19), (44), and the boundedness of $\Omega$, we see that

$$
\begin{gathered}
G_{u}\left(\cdot, u_{n}(\cdot), v_{n}(\cdot)\right), \\
G_{v}\left(\cdot, u_{n}(\cdot), v_{n}(\cdot)\right)
\end{gathered}
$$

$$
\text { are bounded in } L^{2_{*} /\left(2_{*}-1\right)}(\Omega) \text {. }
$$

Since $G(x, u, v)$ is superlinear with respect to $u$ and $v$, we get that the mappings $t \longmapsto G_{u}(x, t, v)$ and $t \longmapsto G_{v}(x, u, t)$ are continuous in $\mathbb{R}$. Therefore,

$$
\begin{gathered}
G_{u}\left(\cdot, u_{n}(\cdot), v_{n}(\cdot)\right) \longrightarrow G_{u}\left(\cdot, u_{\infty}(\cdot), v_{\infty}(\cdot)\right), \\
G_{v}\left(\cdot, u_{n}(\cdot), v_{n}(\cdot)\right) \longrightarrow G_{v}\left(\cdot, u_{\infty}(\cdot), v_{\infty}(\cdot)\right)
\end{gathered}
$$

a.e. in $\Omega$.

Then, by (46) and (47), we have

$$
\begin{aligned}
& G_{u}\left(\cdot, u_{n}(\cdot), v_{n}(\cdot)\right) \rightarrow G_{u}\left(\cdot, u_{\infty}(\cdot), v_{\infty}(\cdot)\right), \\
& G_{v}\left(\cdot, u_{n}(\cdot), v_{n}(\cdot)\right) \rightarrow G_{v}\left(\cdot, u_{\infty}(\cdot), v_{\infty}(\cdot)\right)
\end{aligned}
$$


Noting that $L^{2_{*}}(\Omega)$ is the dual space of $L^{2_{*} /\left(2_{*}-1\right)}(\Omega)$, we deduce that

$$
\begin{aligned}
& \int_{\Omega} G_{u}\left(x, u_{n}, v_{n}\right) \phi \mathrm{d} x \longrightarrow \int_{\Omega} G_{u}\left(x, u_{\infty}, v_{\infty}\right) \phi \mathrm{d} x, \\
& \int_{\Omega} G_{v}\left(x, u_{n}, v_{n}\right) \psi \mathrm{d} x \longrightarrow \int_{\Omega} G_{v}\left(x, u_{\infty}, v_{\infty}\right) \psi \mathrm{d} x
\end{aligned}
$$

for any $\phi, \psi \in L^{2_{*}}(\Omega)$,

and then, in particular,

$$
\begin{aligned}
\int_{\Omega} G_{u}\left(x, u_{n}, v_{n}\right) \phi \mathrm{d} x \rightarrow & \int_{\Omega} G_{u}\left(x, u_{\infty}, v_{\infty}\right) \phi \mathrm{d} x, \\
\int_{\Omega} G_{v}\left(x, u_{n}, v_{n}\right) \psi \mathrm{d} x \longrightarrow & \int_{\Omega} G_{v}\left(x, u_{\infty}, v_{\infty}\right) \psi \mathrm{d} x \\
& \text { for any }(\phi, \psi) \in \mathscr{D}_{K}(\Omega) .
\end{aligned}
$$

For any $(\phi, \psi) \in \mathscr{D}_{K}(\Omega)$, we have

$$
\begin{aligned}
0 & \leftarrow E_{K}^{\prime}\left(u_{n}, v_{n}\right)(\phi, \psi)=\int_{\mathbb{R}^{2 N}}\left(u_{n}(x)-u_{n}(y)\right) \\
& \cdot(\phi(x)-\phi(y)) K(x-y) \mathrm{d} x \mathrm{~d} y \\
& +\int_{\mathbb{R}^{2 N}}\left(v_{n}(x)-v_{n}(y)\right)(\psi(x)-\psi(y)) \\
& \cdot K(x-y) \mathrm{d} x \mathrm{~d} y-\int_{\Omega}\left(\lambda_{1} u_{n} \phi+\lambda_{2} v_{n} \psi\right) \mathrm{d} x \\
& -\int_{\Omega}\left(\mu_{1}\left|u_{n}\right|^{2_{*}-2} u_{n} \phi+\mu_{2}\left|v_{n}\right|^{2_{*}-2} v_{n} \psi\right) \mathrm{d} x \\
& -\int_{\Omega}\left(G_{u}\left(x, u_{n}, v_{n}\right) \phi+G_{v}\left(x, u_{n}, v_{n}\right) \psi\right) \mathrm{d} x .
\end{aligned}
$$

Then, it follows from (43), (45), and (50) that

$$
\begin{aligned}
& \int_{\mathbb{R}^{2 N}}\left(u_{\infty}(x)-u_{\infty}(y)\right)(\phi(x)-\phi(y)) \\
& \cdot K(x-y) \mathrm{d} x \mathrm{~d} y+\int_{\mathbb{R}^{2 N}}\left(v_{\infty}(x)-v_{\infty}(y)\right) \\
& \cdot(\psi(x)-\psi(y)) K(x-y) \mathrm{d} x \mathrm{~d} y \\
& -\int_{\Omega}\left(\lambda_{1} u_{\infty} \phi+\lambda_{2} v_{\infty} \psi\right) \mathrm{d} x \\
& -\int_{\Omega}\left(\mu_{1}\left|u_{\infty}\right|^{2_{*}-2} u_{\infty} \phi+\mu_{2}\left|v_{\infty}\right|^{2_{*}-2} v_{\infty} \psi\right) \mathrm{d} x \\
& -\int_{\Omega}\left(G_{u}\left(x, u_{\infty}, v_{\infty}\right) \phi+G_{v}\left(x, u_{\infty}, v_{\infty}\right) \psi\right) \mathrm{d} x \\
& =0,
\end{aligned}
$$

which means that $\left(u_{\infty}, v_{\infty}\right)$ is solution of (2). It remains to show that $\left(u_{\infty}, v_{\infty}\right)$ is nontrivial. Suppose by contradiction that $\left(u_{\infty}, v_{\infty}\right)=(0,0)$ in $\Omega$. Then, by $(19)$ and boundedness of $\left\{u_{n}\right\}$ and $\left\{v_{n}\right\}$ in $L^{2 *}(\Omega)$, we have

$$
\begin{aligned}
\left|\int_{\Omega} G\left(x, u_{n}, v_{n}\right) \mathrm{d} x\right| \leq & \varepsilon \int_{\Omega}\left(\left|u_{n}\right|^{2 *}+\left|v_{n}\right|^{2 *}\right) \mathrm{d} x \\
& +a(\varepsilon) \int_{\Omega}\left(\left|u_{n}\right|+\left|v_{n}\right|\right) \mathrm{d} x \\
\leq & C \varepsilon+a(\varepsilon)\left(\left|u_{n}\right|_{1, \Omega}+\left|v_{n}\right|_{1, \Omega}\right),
\end{aligned}
$$

and similarly

$$
\begin{aligned}
& \left|\int_{\Omega} G_{u}\left(x, u_{n}, v_{n}\right) u_{n} \mathrm{~d} x\right| \\
& \quad \leq C \varepsilon+a(\varepsilon)\left(\left|u_{n}\right|_{1, \Omega}+\left|v_{n}\right|_{1, \Omega}\right), \\
& \left|\int_{\Omega} G_{v}\left(x, u_{n}, v_{n}\right) v_{n} \mathrm{~d} x\right| \\
& \quad \leq C \varepsilon+a(\varepsilon)\left(\left|u_{n}\right|_{1, \Omega}+\left|v_{n}\right|_{1, \Omega}\right) .
\end{aligned}
$$

Noting that $u_{n} \longrightarrow 0, v_{n} \longrightarrow 0$ in $L^{p}(\Omega)$ for any $p \in\left[1,2_{*}\right)$ and letting $n \longrightarrow \infty$, we get that

$$
\begin{array}{r}
\limsup _{n \rightarrow \infty}\left|\int_{\Omega} G\left(x, u_{n}, v_{n}\right) \mathrm{d} x\right| \leq C \varepsilon, \\
\limsup _{n \rightarrow \infty}\left|\int_{\Omega} G_{u}\left(x, u_{n}, v_{n}\right) u_{n} \mathrm{~d} x\right| \leq C \varepsilon, \\
\limsup _{n \rightarrow \infty}\left|\int_{\Omega} G_{v}\left(x, u_{n}, v_{n}\right) v_{n} \mathrm{~d} x\right| \leq C \varepsilon .
\end{array}
$$

Taking $\varepsilon \longrightarrow 0^{+}$, we have

$$
\begin{gathered}
\int_{\Omega} G\left(x, u_{n}, v_{n}\right) \mathrm{d} x, \\
\int_{\Omega} G_{u}\left(x, u_{n}, v_{n}\right) u_{n} \mathrm{~d} x, \\
\int_{\Omega} G_{v}\left(x, u_{n}, v_{n}\right) v_{n} \mathrm{~d} x
\end{gathered}
$$

0 .

Therefore,

$$
\begin{aligned}
& \left\|\left(u_{n}, v_{n}\right)\right\|_{\mathscr{D}_{K}}^{2}-\mu_{1}\left|u_{n}\right|_{2_{*}, \Omega}^{2_{*}}-\mu_{2}\left|v_{n}\right|_{2_{*}, \Omega}^{2_{*}} \\
& =E_{K}^{\prime}\left(u_{n}, v_{n}\right)\left(u_{n}, v_{n}\right)+\lambda_{1}\left|u_{n}\right|_{2, \Omega}^{2}+\lambda_{2}\left|v_{n}\right|_{2, \Omega}^{2} \\
& \quad+\int_{\Omega} G_{u}\left(x, u_{n}, v_{n}\right) u_{n} \mathrm{~d} x \\
& \quad+\int_{\Omega} G_{v}\left(x, u_{n}, v_{n}\right) v_{n} \mathrm{~d} x \longrightarrow 0 .
\end{aligned}
$$

Assume, up to a subsequence, that $\left\|\left(u_{n}, v_{n}\right)\right\|_{\mathscr{D}_{K}}^{2} \longrightarrow b$. Then, $\mu_{1}\left|u_{n}\right|_{2_{*}, \Omega}^{2_{*}}+\mu_{2}\left|v_{n}\right|_{2_{*}, \Omega}^{2_{*}} \longrightarrow b$, and

$$
B_{K}+o(1)=E_{K}\left(u_{n}, v_{n}\right)=\frac{b}{2}-\frac{b}{2_{*}}+o(1),
$$


which means that $B_{K}=(s / N) b$. By Lemma 5 , we see that $b>0$. Noting (15), we have

$$
\left\|\left(u_{n}, v_{n}\right)\right\|_{\mathscr{D}_{K}}^{2} \geq \bar{S}_{K, \lambda_{1}, \lambda_{2}}\left(\mu_{1}\left|u_{n}\right|_{2_{*}^{*}, \Omega}^{2_{*}}+\mu_{2}\left|v_{n}\right|_{2_{*}, \Omega}^{2_{*}}\right)^{2 / 2_{*}},
$$

which implies that $b \geq S_{K, \lambda_{1}, \lambda_{2}} b^{2 / 2_{*}}$. Therefore, $B_{K} \geq(s /$ $N) S_{K, \lambda_{1}, \lambda_{2}}^{2_{*} /\left(2_{*}-2\right)}=(s / N) S_{K, \lambda_{1}, \lambda_{2}}^{N / 2 s}$, contradicting with Lemma 5. Hence, $\left(u_{\infty}, v_{\infty}\right)$ can not be $(0,0)$. Besides $(45)$, up to a subsequence, we may assume that

$$
\begin{aligned}
& G\left(x, u_{n}, v_{n}\right) \longrightarrow G\left(x, u_{\infty}, v_{\infty}\right) \\
& \text { strongly in } L^{1}(\Omega), \\
& G_{u}\left(x, u_{n}, v_{n}\right) \longrightarrow G_{u}\left(x, u_{\infty}, v_{\infty}\right), \\
& G_{v}\left(x, u_{n}, v_{n}\right) \longrightarrow G_{v}\left(x, u_{\infty}, v_{\infty}\right)
\end{aligned}
$$

strongly in $L^{q /(q-1)}(\Omega)$,

where $q \in\left(2,2_{*}\right)$ is the power sum of $G$ with respect to $u$ and $v$. From

$$
\begin{aligned}
\left\langle u_{n}, \phi\right\rangle_{D_{K}}+\left\langle v_{n}, \psi\right\rangle_{D_{K}}-\lambda_{1} \int_{\Omega} u_{n} \phi \mathrm{d} x & -\lambda_{2} \int_{\Omega} v_{n} \psi \mathrm{d} x-\mu_{1} \int_{\Omega}\left|u_{n}\right|^{2_{*}-2} u_{n} \phi \mathrm{d} x \\
& -\mu_{2} \int_{\Omega}\left|v_{n}\right|^{2_{*}-2} v_{n} \psi \mathrm{d} x \\
& -\int_{\Omega}\left(G_{u}\left(x, u_{n}, v_{n}\right) \phi+G_{v}\left(x, u_{n}, v_{n}\right) \psi\right) \mathrm{d} x \\
= & o(1),
\end{aligned}
$$

we get that

$$
\begin{aligned}
& \left\langle u_{\infty}, \phi\right\rangle_{D_{K}}+\left\langle v_{\infty}, \psi\right\rangle_{D_{K}}-\lambda_{1} \int_{\Omega} u_{\infty} \phi \mathrm{d} x \\
& \quad-\lambda_{2} \int_{\Omega} v_{\infty} \psi \mathrm{d} x \\
& \quad-\mu_{1} \int_{\Omega}\left|u_{\infty}\right|^{2_{*}-2} u_{\infty} \phi \mathrm{d} x-\mu_{2} \int_{\Omega}\left|v_{\infty}\right|^{2_{*}-2} v_{\infty} \psi \mathrm{d} x \\
& \quad-\int_{\Omega}\left(G_{u}\left(x, u_{\infty}, v_{\infty}\right) \phi+G_{v}\left(x, u_{\infty}, v_{\infty}\right) \psi\right) \mathrm{d} x \\
& =0
\end{aligned}
$$

where $(\varphi, \psi) \in \mathscr{D}_{K}(\Omega)$; i.e., $E_{K}^{\prime}(u, v)=0$ in $\mathscr{D}_{K}^{\prime}(\Omega)$. Setting $\omega_{n}=u_{n}-u$ and $\sigma_{n}=v_{n}-v$, then $\omega_{n} \rightarrow 0, \sigma_{n} \rightarrow 0$ weakly in $D_{K}(\Omega)$. Thus, $\omega_{n} \longrightarrow 0, \sigma_{n} \longrightarrow 0$ strongly in $L^{2}(\Omega), \omega_{n} \longrightarrow 0, \sigma_{n} \longrightarrow 0$ a.e. in $\Omega, G_{u}\left(x, \omega_{n}, \sigma_{n}\right) \omega_{n} \longrightarrow 0$, $G_{v}\left(x, \omega_{n}, \sigma_{n}\right) \sigma_{n} \longrightarrow 0, G\left(x, \omega_{n}, \sigma_{n}\right) \longrightarrow 0$ in $L^{1}(\Omega)$, up to a subsequence. Brezis-Lieb Lemma guarantees that

$$
\begin{aligned}
& \left|u_{n}\right|_{2_{*}, \Omega}^{2_{*}}=\left|u_{\infty}\right|_{2_{*}, \Omega}^{2_{*}}+\left|\omega_{n}\right|_{2_{*}^{*}, \Omega}^{2_{*}}+o(1), \\
& \left|v_{n}\right|_{2_{*}, \Omega}^{2_{*}}=\left|v_{\infty}\right|_{2_{*}, \Omega}^{2_{*}}+\left|\sigma_{n}\right|_{2_{*}, \Omega}^{2_{*}}+o(1),
\end{aligned}
$$

$$
\begin{aligned}
\int_{\Omega} G\left(x, u_{n}, v_{n}\right) \mathrm{d} x= & \int_{\Omega} G\left(x, u_{\infty}, v_{\infty}\right) \mathrm{d} x \\
& +\int_{\Omega} G\left(x, \omega_{n}, \sigma_{n}\right) \mathrm{d} x \\
& +o(1) .
\end{aligned}
$$

Since $E_{K}^{\prime}\left(u_{n}, v_{n}\right)\left(u_{n}, 0\right)=o(1), E_{K}^{\prime}\left(u_{n}, v_{n}\right)\left(0, v_{n}\right)=o(1)$ and $E_{K}^{\prime}\left(u_{\infty}, v_{\infty}\right)\left(u_{\infty}, v_{\infty}\right)=0$, by $(63)$, we deduce that

$$
\begin{gathered}
\left\|\omega_{n}\right\|_{D_{K}}^{2}-\mu_{1}\left|\omega_{n}\right|_{2_{*}, \Omega}^{2_{*}}=o(1), \\
\left\|\sigma_{n}\right\|_{D_{K}}^{2}-\mu_{2}\left|\sigma_{n}\right|_{2_{*}, \Omega}^{2_{*}}=o(1),
\end{gathered}
$$

and

$$
I_{K}\left(\omega_{n}, \sigma_{n}\right)=E_{K}\left(u_{n}, v_{n}\right)-E_{K}\left(u_{\infty}, v_{\infty}\right)+o(1),
$$

where

$$
\begin{aligned}
I_{K}(u, v)= & \frac{1}{2}\|(u, v)\|_{\mathscr{D}_{K}}^{2} \\
& -\frac{1}{2_{*}}\left(\mu_{1}|u|_{2_{*}, \mathbb{R}^{N}}^{2_{*}}+\mu_{2}|v|_{2_{*}, \mathbb{R}^{N}}^{2_{*}}\right)
\end{aligned}
$$

Assume that

$$
\begin{aligned}
& \lim _{n \longrightarrow \infty} \mu_{1}\left|\omega_{n}\right|_{2_{*}, \Omega}^{2_{*}}=a_{1}, \\
& \lim _{n \longrightarrow \infty} \mu_{2}\left|\sigma_{n}\right|_{2_{*}, \Omega}^{2_{*}}=a_{2},
\end{aligned}
$$

up to a subsequence. By (64), we see that

$$
I_{K}\left(\omega_{n}, \sigma_{n}\right)=\left(\frac{1}{2}-\frac{1}{2_{*}}\right)\left(a_{1}+a_{2}\right)+o(1) .
$$

Letting $n \longrightarrow \infty$, we have

$$
\begin{aligned}
B_{K} & =\lim _{n \longrightarrow \infty} E_{K}\left(u_{n}, v_{n}\right) \\
& =\left(\frac{1}{2}-\frac{1}{2_{*}}\right)\left(a_{1}+a_{2}\right)+E_{K}\left(u_{\infty}, v_{\infty}\right) \geq 0 .
\end{aligned}
$$

Suppose by contradiction that $\left(u_{\infty}, v_{\infty}\right)=\left(u_{\infty}, 0\right)$, where $u_{\infty} \not \equiv 0$. Then, $u_{\infty}$ is a nontrivial solution of $\mathscr{L}_{K} u+$ $\lambda_{1} u+\mu_{1}|u|^{2_{*}-2} u=0$. Hence, by (69), we deduce that $B_{K} \geq$ $E_{K}\left(u_{\infty}, 0\right) \geq(s / N) S_{K, \lambda_{1}}^{N / 2 s} \geq(s / N) \bar{S}_{K, \lambda_{1}, \lambda_{2}}^{N / 2 s}$, contradiction with Lemma 5. Therefore, $\left(u_{\infty}, v_{\infty}\right)$ can not be $\left(u_{\infty}, 0\right)$, where

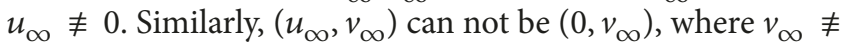
0 . Thus, (2) has a nontrivial ground state solution, which completes the proof.

\section{Data Availability}

No data were used to support this study.

\section{Conflicts of Interest}

The authors declare that they have no conflicts of interest. 


\section{Acknowledgments}

This work is supported by NSFC (11701248).

\section{References}

[1] E. Di Nezza, G. Palatucci, and E. Valdinoci, "Hitchhiker's guide to the fractional Sobolev spaces," Bulletin des Sciences mathématiques, vol. 136, no. 5, pp. 521-573, 2012.

[2] G. M. Bisci, V. D. Radulescu, and R. Servadei, Variational Methods for Nonlocal Fractional Problems, vol. 162, Cambridge University Press, 2016.

[3] R. Servadei and E. Valdinoci, "The Brezis-Nirenberg result for the fractional Laplacian," Transactions of the American Mathematical Society, vol. 367, no. 1, pp. 67-102, 2015.

[4] Z. Chen and W. Zou, "Positive least energy solutions and phase separation for coupled Schrödinger equations with critical exponent," Archive for Rational Mechanics and Analysis, vol. 205, no. 2, pp. 515-551, 2012.

[5] Z. Chen and W. Zou, "Positive least energy solutions and phase separation for coupled Schrödinger equations with critical exponent: higher dimensional case," Calculus of Variations and Partial Differential Equations, vol. 52, no. 1-2, pp. 423-467, 2015.

[6] R. Servadei and E. Valdinoci, "Mountain pass solutions for nonlocal elliptic operators," Journal of Mathematical Analysis and Applications, vol. 389, no. 2, pp. 887-898, 2012.

[7] A. Ambrosetti and P. H. Rabinowitz, "Dual variational methods in critical point theory and applications," Journal of Functional Analysis, vol. 14, pp. 349-381, 1973.

[8] H. Brezis and L. Nirenberg, "Positive solutions of nonlinear elliptic equations involving critical Sobolev exponents," Communications on Pure and Applied Mathematics, vol. 36, no. 4, pp. $437-477,1983$. 


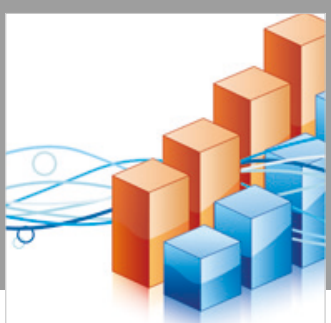

Advances in

Operations Research

\section{-n-m}
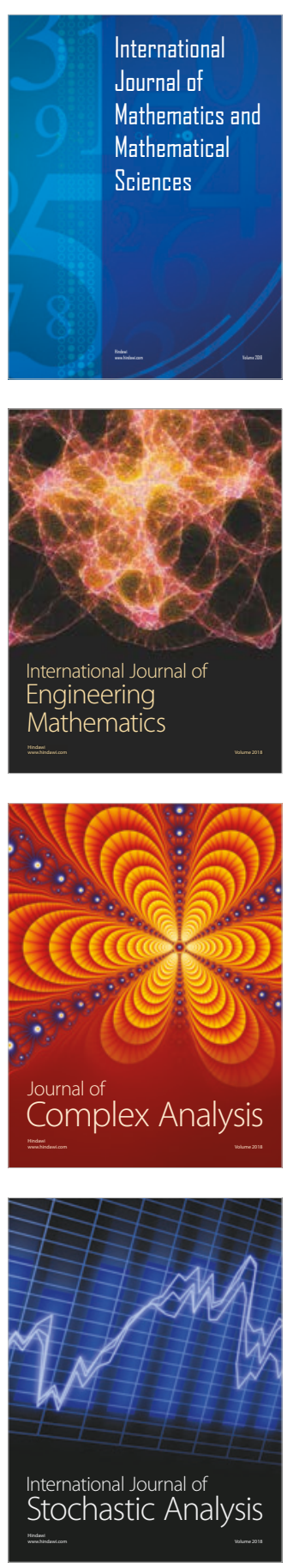
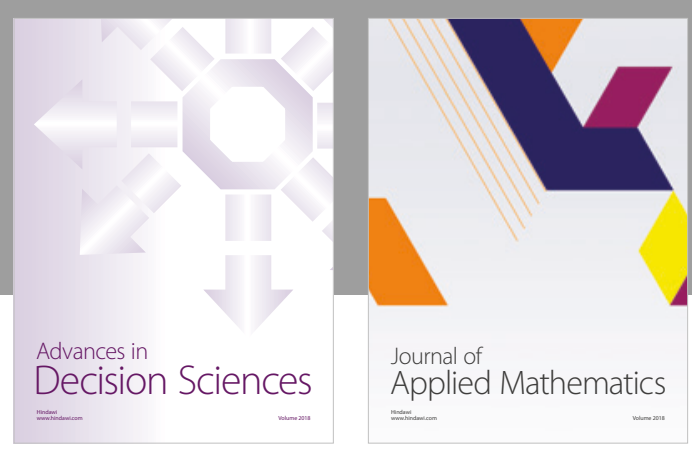

Journal of

Applied Mathematics
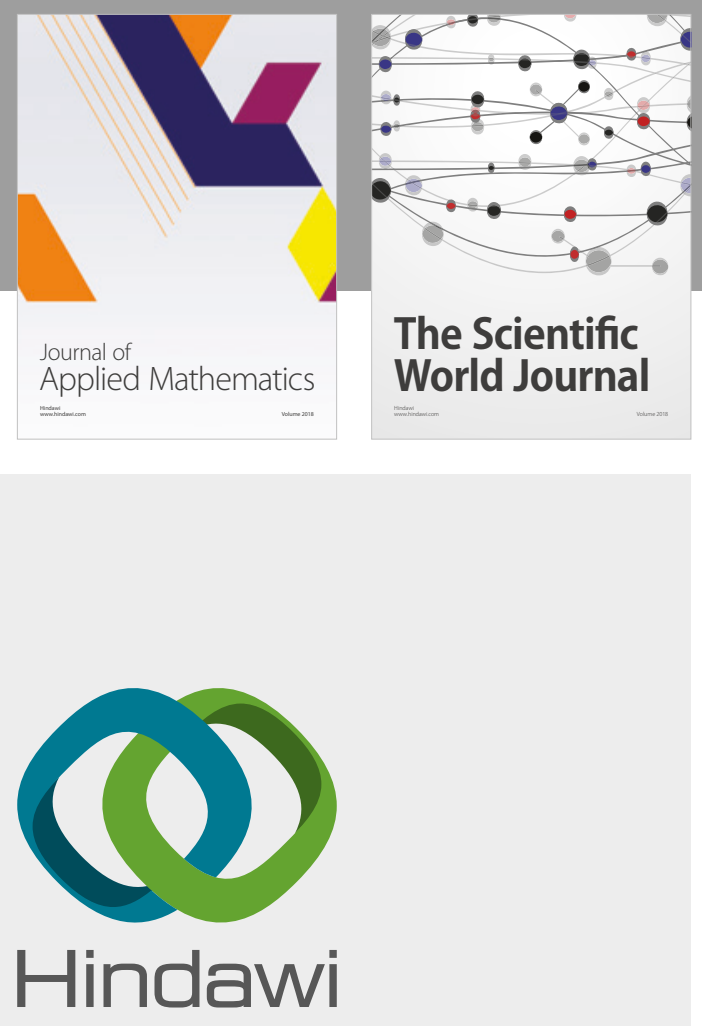

Submit your manuscripts at

www.hindawi.com

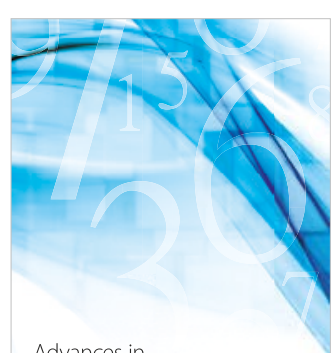

Advances in
Numerical Analysis
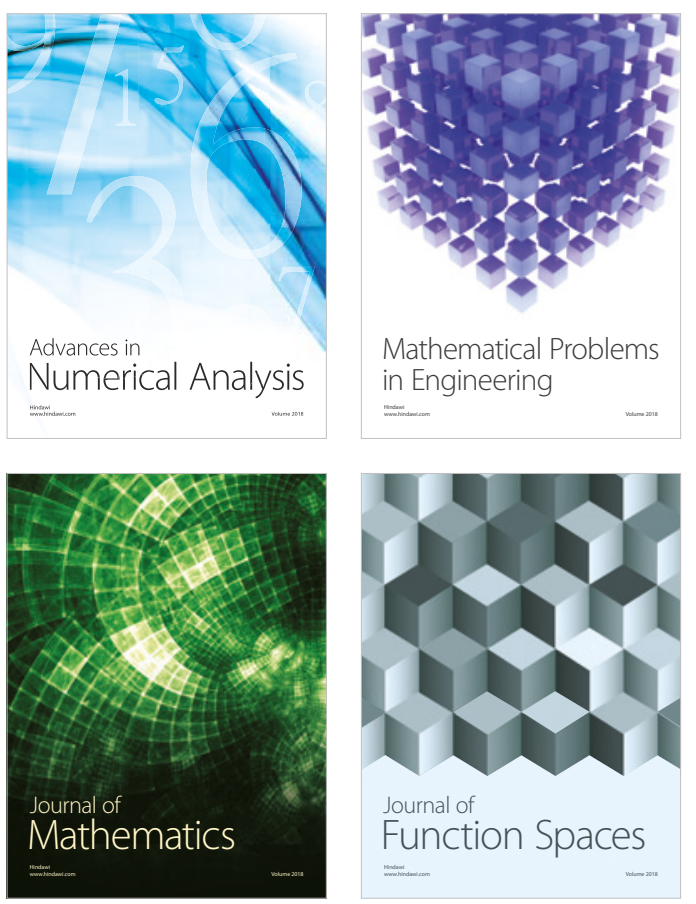

Mathematical Problems in Engineering

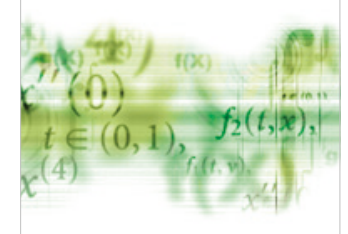

International Journal of

Differential Equations

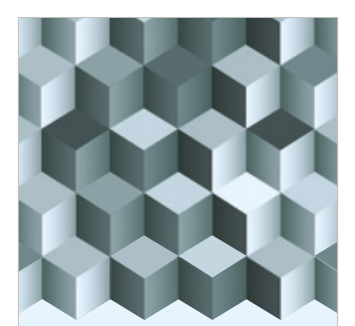

Journal of

Function Spaces

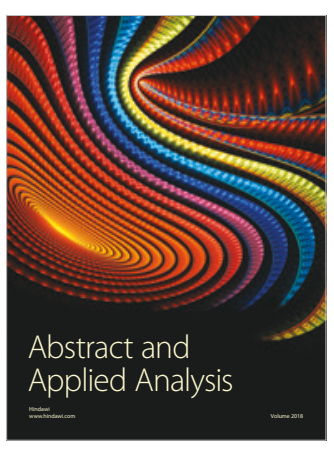

The Scientific

World Journal

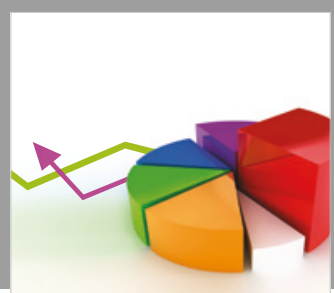

Journal of

Probability and Statistics
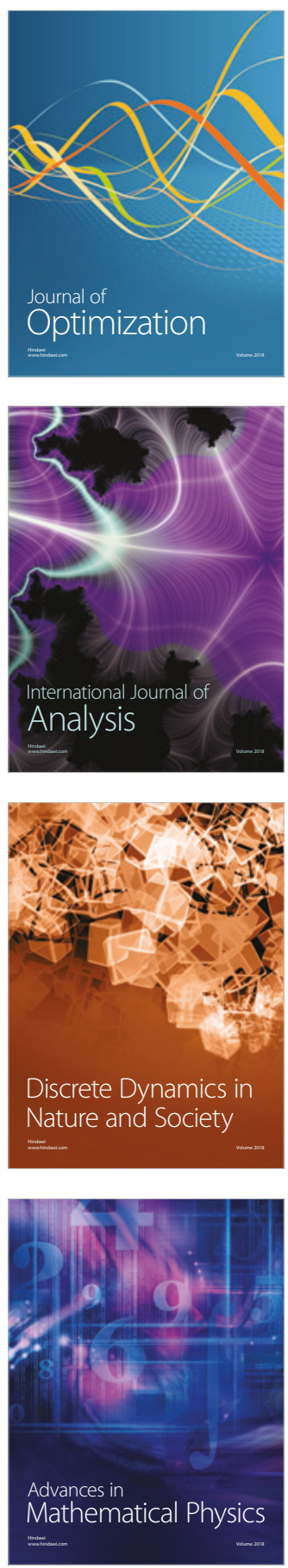